Psychotherapeut 2018 63:271-282

DOI 10.1007/s00278-017-0214-8

Online publiziert: 3. August 2017

(c) Der/die Autor(en) 2017. Dieser Artikel ist eine Open-Access-Publikation.

\section{Redaktion}

W. Schneider, Rostock

T. Fydrich, Berlin

CrossMark

\author{
Raphael Schuster ${ }^{1}$ Thomas Berger ${ }^{2} \cdot$ Anton-Rupert Laireiter $^{1,3}$ \\ ${ }^{1}$ Fachbereich Psychologie, Universität Salzburg, Salzburg, Österreich \\ ${ }^{2}$ Institut für Klinische Psychologie und Psychotherapie, Universität Bern, Bern, Schweiz \\ ${ }^{3}$ Fakultät für Psychologie, Universität Wien, Wien, Österreich
}

\title{
Computer und Psychotherapie - geht das zusammen?
}

\section{Stand der Entwicklung von Online- und gemischten Interventionen in der Psychotherapie}

rung internetbasierter Interventionen auswirken.

\section{Die Entwicklung moderner Kom- munikationsmittel verzeichnet ein ungebremstes Wachstum und eine fortschreitende Ausdifferenzierung hinsichtlich möglicher Anwendungs- gebiete. Dieser Trend wirkt sich zunehmend auch auf den psychoso- zialen Sektor und hier insbesondere auf die Psychotherapie aus. Diese Entwicklung hat mittlerweile auch den deutschen Sprachraum erreicht.}

\section{Hintergrund}

Von besonderer Bedeutung sind verschiedene Formen computerbasierter Interventionen, die Gegenstand der vorliegenden Arbeit sind. Berger und Caspar (2011) nennen folgende Gründe für diese Entwicklung:

- technologischer Fortschritt als Basis für günstige und leistungsfähige internetbasierte Interventionen,

- gesellschaftliche Entwicklung im Sinne einer Anpassung und Gewöhnung an neue Medien,

- Nachfrage nach psychosozialer Unterstützung, die durch klassische Angebote nicht in vollem Umfang abgedeckt wird,

- Spezifizierung und Standardisierung psychotherapeutischer Methoden, die sich gut in internetbasierte Beratungsangebote übersetzen lassen,

- vielversprechende Wirksamkeitsnachweise, die sich förderlich auf die Beforschung und die Implementie-

\section{auswirken.}

\section{Begriffsklärung: internet- basierte Interventionen und gemischte Psychotherapie}

Der Auftakt zur Entwicklung computerisierter Psychotherapie erfolgte bereits in den 1960er-Jahren mit dem Programm „Eliza“ (Weizenbaum 1966). Ursprünglich verfolgte Weizenbaum mit der Simulation des Kommunikationsverhaltens eines humanistischen Psychotherapeuten genau das umgekehrte Ziel. Er wollte zeigen, dass menschliche Kommunikation nicht durch ein Computerprogramm ersetzt werden kann. Fünfzig Jahre später wird seine Arbeit unter ähnlichem Namen („Ellie“) und umgekehrtem Motiv fortgeführt. Bei Ellie handelt es sich um einen virtuellen Avatar, der menschliche Kommunikation mithilfe einer Stimm- und Gesichtsanalyse in Echtzeit bestmöglich imitieren soll (Robinson 2015). Während die angewandte Grundlagenforschung also weiter an der psychotherapeutischen Mensch-Computer-Schnittstelle bastelt, versucht die „Online-Behandlung“ der Gegenwart nicht, Therapeuten bestmöglich nachzuahmen. Vielmehr lässt sie sich als interaktive, multimediagestützte Bibliotherapie (Watkins 2008) oder Selbsthilfe 2.0 beschreiben. Obgleich eine eindeutige Begriffsbestimmung durch die rasche Entwicklung des Forschungsgebietes erschwert wird (Barak et al. 2009), beschreiben Barak et al. (2009, S. 5) OnlineInterventionen (syn. internetbasierte Interventionen, Internet-Interventionen oder computerbasierte Interventionen) als: „... vordergründig selbstgesteuerte Interventionsprogramme, welche einem festgelegten Behandlungsprotokoll folgen, über einen Onlinedienst bereitgestellt und von Endnutzern zur Unterstützung bei psychischen oder gesundheitsbezogenen Problemen verwendet werden ...". Dabei lässt sich die Intensität der einzelnen Programme über drei Stufen beschreiben, in denen rein psychoedukative oder informative Internetseiten (als unterste Stufe) von selbstgesteuerten Online-Trainings mit Selbsthilfecharakter (mittlere Stufe) gefolgt werden (Andersson 2014, S. 2). Auf der höchsten Intensitätsstufe werden Online-Interventionen durch Therapeuten oder geschultes Personal per E-Mail- oder Telefonkontakt flankiert. Wöchentlich werden pro Klient in der Regel 10-15 min Betreuungszeit veranschlagt (Andersson et al. 2014), wenngleich manche Programme umfangreichere Betreuung voraussetzen. Online-Interventionen können somit also als „Stand-alone“-Programme bis hin $\mathrm{zu}$ einem Adjunkt zur klassischen Psychotherapie eingesetzt werden. Letzteres kommt dem zweiten Begriff dieser Arbeit sehr nahe („blended therapy“).

Gemischte Psychotherapie („blended therapy“ oder „blended interventions“) 
baut auf dem persönlichen Kontakt zwischen Therapeuten und Patienten und dem klassischen Therapiesetting auf, versucht dieses in den verschiedenen Phasen des Therapieprozesses um Bestandteile internetbasierter Interventionen $\mathrm{zu}$ erweitern und daraus ein integratives Behandlungskonzept abzuleiten (Riper et al. 2013). Das deklarierte Ziel gemischter Psychotherapie ist es, das Beste aus beiden Welten vereinen zu wollen (Ruwaard und Kok 2015). Vorteile gemischter Psychotherapie liegen in der Steigerung der Behandlungsdosis sowie der Förderung der Mitarbeit des Patienten und der Übertragung des Gelernten in den Alltag (Kooistra et al. 2016). Damit verbunden ist in der Regel eine Reduktion des therapeutischen („Faceto-face"-)Aufwands. Entsprechend können gemischte Psychotherapien darauf abzielen, die Therapiezeit im persönlichen Kontakt zu reduzieren und dabei vergleichbare Therapieergebnisse zu erreichen (Wright et al. 2005).

Sowohl internetbasierte als auch gemischte Interventionen sind in ihrer Beschaffenheit von der "Telemedizin“ (etwa Chat-Gruppen oder Telefontherapie) sowie der „virtuellen Realität" (etwa computersimulierte Konfrontation bei Höhenangst oder Tierphobien) und ,serious gaming" abzugrenzen. In der Telemedi$z$ in werden meist klassische Leistungen auf dem Weg neuer Medien überbracht; in der virtuellen Realität werden einzelne spezifische Behandlungstechniken (z. B. Expositionen) in die digitale Welt übersetzt und "serious gaming" beinhaltet eine Reihe heterogener Ansätze mit vergleichbarer Zielsetzung, wie z. B. Lernspiele, Fahrt- oder Flugsimulationen etc. Online- und gemischte Psychotherapien hingegen sind sehr viel stärker auf den Therapieprozess hin abgestimmt bzw. in diesen eingepasst und erweitern ihn um jeweils neue Aspekte.

\section{Inhalte von Online-Inter- ventionen und gemischter Psychotherapie}

Die Inhalte internetbasierter und gemischter Interventionen können sich je nach Störungsbild und Behandlungsstrategie sehr ähnlich sein oder sich auch deutlich voneinander unterscheiden. Das Spektrum reicht von methodisch sparsamen Ansätzen (wie der Verhaltensaktivierung; Lejuez et al. 2001) bis hin zu umfassenden und integrativen Ansätzen. Weil beide Behandlungsformen einen gemeinsamen Ursprung besitzen, ähneln sich viele der eingesetzten Behandlungsbestandteile. So stellen etwa elektronische Tagebücher, interaktive Textaufgaben sowie multimediagestützte Psychoedukation oder Fallbeispiele typische Bestandteile beider Interventionsformen dar.

Wenngleich psychodynamisch- oder interpersonell-orientierte Ansätze existieren (Andersson und Titov 2014; Johansson et al. 2012), basieren die meisten der bisher entwickelten Interventionen auf Prinzipien der kognitiven Verhaltenstherapie. In der Regel bestehen ihre computerbasierten Komponenten aus einer bestimmten Abfolge thematisch abgrenzbarer Module, die über eine Online-Plattform chronologisch oder entsprechend dem persönlichen Interesse bearbeitet werden können. In angloamerikanischen, holländischen und skandinavischen Ländern existieren bereits sehr viele derartige Ansätze (Hedman et al. 2012). Als Beispiel aus dem deutschen Sprachraum soll an dieser Stelle der Inhalt einer schulenübergreifenden Online-Intervention für Depression dargestellt werden (Deprexis; www.deprexis.com; Meyer et al. 2009). Diese besteht insgesamt aus 10 Modulen, die entsprechend den individuellen Bedürfnissen und Wünschen des Patienten bearbeitet werden können: (1) Verhaltensaktivierung, (2) kognitive Umstrukturierung, (3) Achtsamkeit und Akzeptanz, (4) interpersonelle Kompetenzen, (5) Entspannung, körperliche Betätigung und Lebensstil, (6) Problemlösekompetenzen, (7) Kindheit und frühe Lebensschemata, (8) positive Psychologie, (9) traum- und emotionsfokussierte Techniken sowie (10) Psychoedukation. Um das jeweils passende Modul auszuwählen, leitet ein Computeralgorithmus auf Basis eines simulierten textbasierten Dialogs entsprechende Vorschläge ab. Auf diese Weise sollen die Inhalte des Programms bestmöglich an individuelle Bedürfnisse angepasst und dynamisch präsentiert werden. Sowohl für den Symptomverlauf als auch für die Bearbeitung der Module können Statistiken und $\mathrm{Zu}$ sammenfassungen abgefragt werden. Optional sendet das Programm unterstützende Erinnerungsnachrichten auf das Mobiltelefon, um den Lerntransfer $\mathrm{zu}$ unterstützen. Weil das Programm sowohl als Adjunkt klassischer Psychotherapie eingesetzt werden kann (Krieger et al. 2014) wie auch als reine OnlineIntervention, ist es in seiner Anwendung an der Grenze zwischen internetbasierter Intervention und gemischter Psychotherapie anzusiedeln. Neben textbasierten Interventionsbestandteilen kommen je nach Programm Audiomaterialien, Videoclips oder Fallbespiele zum Einsatz, die interaktiv bearbeitet werden können und die Attraktivität und Vielseitigkeit der Anwendungen verbessern sollen (etwa: Lin et al. 2014; Eells et al. 2014).

\section{Wirksamkeit von Online- Interventionen}

Im Zeitraum von 2000 bis 2012 wurden Online-Interventionen in über 100 randomisierten kontrollierten Studien („randomized controlled trial“, RCT) für 25 unterschiedliche klinische Beeinträchtigungen beforscht (Hedman et al. 2012). Die Wirksamkeit bei Depressionen, sozialer Phobie und Panikstörungen kann als empirisch nachgewiesen angesehen werden. Weitere Studien liegen u. a. für Essstörungen (Carrard et al. 2011), pathologisches Glücksspiel (Carlbring und Smit 2008), komplizierte Trauer (Wagner et al. 2006), stoffgebundene Abhängigkeiten (Tossmann et al. 2011) und posttraumatische Belastungsstörungen (Kuester et al. 2016) sowie für funktionelle Störungen (wie etwa Tinnitus, chronischer Schmerz oder Fibromyalgie; Hedman et al. 2012) vor. Zudem gibt es bereits erste Online-Kliniken, wie etwa das „Black Dog Institute“ in Sydney, Australien, oder die psychiatrische Internet-Abteilung am KarolinskaUniversitätskrankenhaus in Stockholm. Allein in Schweden wurden innerhalb von 5 Jahren 1200 depressive Menschen routinemäßig mit internetbasierten Interventionen behandelt (Hedman et al. 2014). 
Die Behandlungsergebnisse internetbasierter Interventionen können sich durchaus sehen lassen. Für Depression betragen die berichteten Effektstärken aus 5 Metaanalysen zwischen $d=0,41$ und $d=0,94$ (Andrews und Williams 2014), und Online-Programme für Angststörungen weisen im Schnitt eine Symptomreduktion von $d=0,92$ auf (Adelman et al. 2014). Die gefundenen Effekte sind meist vom Schweregrad der Beeinträchtigung sowie vom Umfang der gleichzeitig gegebenen therapeutischen Unterstützung (Johansson und Andersson 2012) abhängig. Während die Veränderungsmessung in vielen Studien auf Selbstbeurteilungsverfahren beruht, wurden in einigen Studien ebenfalls positive Effekte in der Fremdbeurteilung berichtet (Ebert et al. 2014; Klein et al. 2016). Zusätzlich liegen Ergebnisse aus bildgebenden Verfahren vor, die belegen, dass Onlinebehandlungen $\mathrm{zu}$ gleichen neuronalen Veränderungen führen, wie sie auch nach erfolgreicher klassischer Psychotherapie beobachtet werden (Månsson et al. 2016).

In den letzten Jahren wurde zunehmend der direkte Vergleich zwischen klassischer Psychotherapie und OnlineInterventionen angetreten. Programme, die von Therapeuten online unterstützend begleitet werden, wiesen in einer 13 Studien umfassenden Metaanalyse gleiche Effekte auf, wie klassische Einzeloder Gruppenpsychotherapie (Andersson et al. 2014). Dabei beurteilen die Autoren sowohl die Qualität als auch die Heterogenität der eingeschlossenen Studien als hoch.

\section{Wer profitiert von Online- Interventionen?}

Befunde zur differenziellen Wirksamkeit von internetbasierten Interventionen weisen bisher über weite Strecken niedrige Konsistenz auf. Neben grundlegenden Voraussetzungen wie Internetzugang und guten Computerkenntnissen (Waller und Gilbody 2009; Gerhards et al. 2011) sowie persönlichem Interesse und individuellem Hilfesuchverhalten (Gieselmann et al. 2015; Eichenberg et al. 2013) wurden Ressourcen wie die Übertragung der Trainingsinhalte in den per-

Psychotherapeut 2018 63:271-282 DOI 10.1007/s00278-017-0214-8

(c) Der/die Autor(en) 2017. Dieser Artikel ist eine Open-Access-Publikation.

\section{R. Schuster $\cdot$ T. Berger $\cdot$ A.-R. Laireiter}

\section{Computer und Psychotherapie - geht das zusammen? Stand der Entwicklung von Online- und gemischten Interventionen in der Psychotherapie}

\section{Zusammenfassung}

Die Verbreitung moderner Medien wirkt sich zunehmend auf psychologische Behandlungsangebote aus. Während die empirische Basis internetbasierter Interventionen zur Behandlung häufiger psychischer Beeinträchtigungen wächst und ihre Anwendung in der Regelversorgung gegenwärtig international erprobt wird, bestehen zusätzliche Bestrebungen, das gewonnene Knowhow mit konventioneller Psychotherapie zu kombinieren. Die daraus entstehende Behandlungsform der "gemischten Psychotherapie“ („,blended therapy") hat das deklarierte Ziel, das Beste aus beiden Welten zu vereinen. Nach einer einleitenden Begriffsbestimmung gibt die vorliegende Übersichtsarbeit Einblick in die Inhalte und die Bestandteile internetbasierter sowie gemischter Interventionen. Der aktuelle
Forschungsstand wird skizziert und vor dem Hintergrund der praktischen Anwendbarkeit durch eine Zusammenschau quantitativer und qualitativer Studien dargelegt. Besonderes Augenmerk gilt der Rolle der therapeutischen Unterstützung und der Beschaffenheit der therapeutischen Allianz bei internetbasierten Interventionen sowie den Stärken und Schwächen beider Behandlungsstrategien. Ziel ist es, sowohl die Entwicklung als auch das Potenzial beider Forschungsrichtungen plausibel und realistisch darzustellen und in eine strategische Versorgungsperspektive zu setzen.

\section{Schlüsselwörter}

E-Mental-Health · Gemischte Therapie · Online Intervention · Psychotherapie - Psychische Gesundheitsversorgung

\section{Computer and psychotherapy - do they fit? Review of the state of development of internet-based and blended interventions in psychotherapy}

\section{Abstract}

Modern media have an increasing impact on psychological health services. While the empirical foundation of internet-based interventions for frequently occurring mental disorders grows and their effectiveness is investigated in routine care trials, efforts also exist to transfer the knowledge gained to classical psychotherapy. The resulting treatment format "blended therapy" aims to combine the best of both worlds. After an introductory definition of both terms this overview describes the contents and components of internet-based and blended interventions. Summarizing the current state of research with an emphasis on feasibility,

selected quantitative and qualitative articles are presented. The article depicts the development, application and potential of both interventional strategies and focuses on the role of therapist support as well as on the nature of the therapeutic alliance. Strengths and weaknesses of each intervention are addressed and integrated into a strategic perspective.

\section{Keywords}

E-mental health · Blended therapy · Online intervention · Psychotherapy · Mental health care

sönlichen Alltag mit besseren Erfolgen in Verbindung gebracht. Hierzu zählen etwa frei verfügbare Zeit oder soziale Unterstützung aus dem näheren Umfeld (Gerhards et al. 2011; Donkin und Glozier 2012; Farrer et al. 2013). In manchen Studien stehen Faktoren wie Therapiemotivation, Erfolgserwartungen sowie strukturiertes und diszipliniertes Arbeiten mit positiven Ergebnissen im
Zusammenhang (Donkin und Glozier 2012; Ebert et al. 2013a; Bendelin et al. 2011). Hinsichtlich Geschlecht, Symptomschwere und Persönlichkeit ist die Befundlage wenig eindeutig (Andersson et al. 2014). Wenngleich bisher keine allgemeinen Entscheidungsregeln für den individuellen Einsatz von OnlineProgrammen festgelegt werden konnten, bieten erste Checklisten eine praktische 
Entscheidungshilfe bei der Indikationseinschätzung (Berger 2015; Wentzel et al. 2016).

\section{Rolle der therapeutischen Unterstützung und der therapeutischen Allianz bei Online-Interventionen}

Onlineinterventionen werden als Standalone-Programme oder mit therapeutischer Begleitung angeboten. Während Stand-alone-Programme mit niedrigeren Kosten verbunden sind, führen begleitete Online-Interventionen meist $\mathrm{zu}$ besseren Ergebnissen. Dieser Effekt tritt deutlich bei Programmen zur Reduktion der Depression zutage. Hier betrug die Symptomreduktion für Standalone-Programme (ohne diagnostischen Patientenkontakt) in einer aktuellen Metaanalyse $d=0,21$. Der Gruppeneffekt für Interventionen mit persönlicher diagnostischer Abklärung plus laufender Unterstützung belief sich hingegen auf $d=0,76$ (Johansson und Andersson 2012). Unbegleitete Online-Interventionen weisen zudem häufig problematisch hohe Abbruchquoten auf. Auch hier wirkt persönlicher Kontakt förderlich und kann das Risiko eines vorzeitigen Behandlungsabbruchs, einer Metaanalyse von Richards und Richardson (2012) zufolge, um das bis zu Siebenfache („odds ratio" $[\mathrm{OR}]=7,35$ ) reduzieren. Diese Befunde sind jedoch nicht genereller Natur, sondern müssen vor dem Hintergrund der spezifischen Störung betrachtet werden. So scheinen die berichteten Ergebnisse beispielsweise nicht auf die internetbasierte Behandlung von Angststörungen übertragbar zu sein (Berger et al. 2011; Titov et al. 2015). Die therapeutische Begleitung basiert bei Online-Interventionen meist auf zeitnaher asynchroner E-Mail-Korrespondenz. Neben der Unterstützung bei technischen Belangen zielt Therapeutenkontakt insbesondere darauf $a b$, die Compliance bei der Bearbeitung der Module zu fördern (Setzen von Erinnerungen, positive Verstärkung etc.), die Selbstwirksamkeit der Klienten und die therapeutische Allianz zu stärken sowie Empathie und Anteilnahme auszudrücken (Paxling et al. 2013).
Das Konzept der therapeutischen Allianz wird in der Psychotherapieforschung weithin als wichtiger unspezifischer Wirkfaktor angesehen (Lambert und Barley 2002; Orlinsky et al. 2004). Daraus ergibt sich die Frage, wie die therapeutische Allianz von Klienten im Online-Setting wahrgenommen wird. Obwohl sich Klient und Therapeut meist nicht persönlich kennen, in der Interaktion auf nonverbale Kommunikation verzichten müssen und der Umfang des Kontaktes zeitlich deutlich reduziert ist, findet ein Austausch über das Privatleben des Klienten und somit Selbstöffnung statt. Zusätzlich werden gemeinsam Aufgaben und Ziele der Therapie formuliert und verfolgt. Faktoren, die sich durch das besondere Setting positiv auf die Einschätzung der therapeutischen Beziehung auswirken könnten, sind zudem der Enthemmungseffekt mittelbarer Kommunikation (Suler 2004) sowie der Umstand, dass mögliche Negativmerkmale der Persönlichkeit und des Auftretens behandelnder Therapeuten weniger deutlich zur Geltung kommen (Andersson et al. 2012). In den meisten Studien wird die therapeutische Allianz im Online-Setting (oft auf Basis des Working Alliance Inventory, WAI, von Horvath und Greenberg 1989) als gut beschrieben und ist damit - möchte man der Argumentationslinie mancher Befürworter folgen - der therapeutischen Allianz klassischer Psychotherapie gleichzustellen. Obwohl einige Studien versuchten, die therapeutische Allianz beider Interventionsformen direkt $\mathrm{zu}$ vergleichen (Preschl et al. 2011), besitzen die Teilnehmer der jeweiligen Versuchsbedingung lediglich Erfahrung mit nur einer der beiden Interventionsarten, weshalb unterschiedliche Referenzpunkte der Teilnehmer die Vergleichbarkeit der Ergebnisse einschränken. Die Einschätzung der therapeutischen Allianz besitzt zudem, in Abhängigkeit von der jeweiligen Studie, unterschiedliche Vorhersagekraft hinsichtlich des zu erwartenden Therapieerfolgs (Knaevelsrud und Maercker 2006; Bergman et al. 2013; Berger 2016).

Insgesamt und zusammenfassend ist also davon auszugehen, dass therapeutische Unterstützung ein wesentlicher Erfolgsfaktor bei Online-Programmen ist. Während die therapeutische Beziehung im Online-Setting inhaltlich und strukturell deutliche Unterschiede zur herkömmlichen Face-to-face-Therapie aufweist, wird sie von Klienten, sowohl hinsichtlich der Aufgaben- und Zielorientierung als auch hinsichtlich der wahrgenommenen Bindung, in der Regel als vergleichbar intensiv und hilfreich beschrieben. Die Annahme, es handle sich in beiden Therapieformen qualitativ um dieselbe Art, erscheint sowohl aufgrund logischer Überlegungen, v. a. aber aufgrund der verwendeten Methoden und der unterschiedlichen Bezugspunkte bei der Einschätzung der Allianz (Berger 2016) als eher unwahrscheinlich.

\section{Einschränkungen von Online- Interventionen}

Neben der guten Reichweite, der für viele Störungen gut belegten Wirkung sowie ihrer Flexibilität weisen OnlineInterventionen aber auch Nachteile auf verschiedenen Ebenen auf. Eine qualitative Diagnostik wird etwa durch den reduzierten Patient-Therapeut-Kontakt erschwert, und es bestehen gegenwärtig Einschränkungen hinsichtlich der Anwendbarkeit bei Komorbiditäten (Andersson und Titov 2014). Wenngleich manche Studien die Wirksamkeit von Online-Interventionen bei schwereren Krankheitsverläufen belegen (Meyer et al. 2015), bestehen praktische Einschränkungen hinsichtlich der Abfederung möglicher Krisen oder bei Suizidalität. Auf der Versorgungsebene sind hohe Abbruchquoten und bessere Wirksamkeit bei therapeutischer Unterstützung sowie Vorbehalte seitens mancher betroffenen Akteure des Gesundheitswesens als einschränkende Faktoren $\mathrm{zu}$ nennen (Andersson und Titov 2014; Tarrier et al. 2006). Eine rechtliche Grenze im Einsatz von internetbasierten Interventionen besteht mit dem in Kraft befindlichen Fernbehandlungsverbot (Bauer und Kordy 2008). In - Tab. 1 sind ausgewählte Vor- und Nachteile von Online-Interventionen zusammengefasst. 
Tab. 1 Vor- und Nachteile von Online-Interventionen

\begin{tabular}{l} 
Vorteile \\
\hline Überbrücken von Distanzen \\
Gute Reichweite und Skalierbarkeit (im Si \\
eines guten versorgungstechnischen „up \\
ling") \\
Anpassbarkeit an manche Patientenbedü \\
nisse möglich (etwa flexibler Abruf der In \\
te, Anonymität) \\
Therapeutische Begleitung möglich und \\
förderlich \\
Wirksamkeit bei gewissen Beeinträchtigun- \\
gen bereits gut belegt \\
Schnelle Hilfe und Behandlung während \\
Wartezeit möglich \\
Gemischte Psycho- \\
therapie - ein anwendbares \\
Behandlungskonzept?
\end{tabular}

Die Beforschung von Online- und gemischter Psychotherapie („,blended therapy“) kann - gemeinsam mit der Forschung im Bereich der virtuellen Realität - bereits auf die späten 1980er- bzw. frühen 1990er-Jahre datiert werden. Der unterstützende Einsatz von Computerprogrammen als Adjunkt klassischer Psychotherapie wurde so bereits früh bei der Anwendung kognitiv-behavioraler Techniken, wie etwa Entspannungsübungen, systematischer Desensibilisierung, kognitiver Umstrukturierung oder Expositionsübungen, untersucht (u. a. Baer und Surman 1985; Newman et al. 1996). Weil die wenigsten Haushalte zu dieser Zeit Computer besaßen und das Internet lediglich zu Forschungszwecken genutzt wurde, kamen in der damaligen computerunterstützten Psychotherapie (oftmals als „computer-assisted therapy“ oder „computer-supported therapy“ bezeichnet) primär Taschencomputer mit Mehrzeilendisplay zum Einsatz. Die daraus entstehenden Anwendungsmöglichkeiten sind aus heutiger Sicht als sehr eingeschränkt zu bezeichnen. Mit der Rechenkapazität moderner Computer und dem anhaltenden Siegeszug des World Wide Web verbessern sich gegenwärtig die Einsatzmöglichkeiten moderner Medien in der Psychotherapie. So besitzen Smartphones vielseitige Funktionalität, die Handhabung derselben wurde von ihren Anwendern

\section{Nachteile}

Erschwerte Diagnostik und eingeschränkte Unterstützung bei Krisen

Nicht für alle Patienten geeignet (Einschränkungen bei Komorbiditäten und schweren Beeinträchtigungen)

Anpassbarkeit an manche Patientenbedürfnisse eingeschränkt (etwa Bedürfnis nach persönlichem Kontakt)

Risiko von Fehlanwendungen und hohe $\mathrm{Ab}$ bruchquote

Bedenken seitens mancher Akteure im Gesundheitssystem

Rechtliche Einschränkungen bei Anwendung vorhanden

gut internalisiert, und Taschencomputer haben nach ihrer Transformation zu Tablets und iPads endgültig den Markt erobert. E-Learning-Plattformen, wie Moodle, tragen diesem Trend Rechnung und bieten ihre Software-Dienstleistungen entsprechend synchron für Laptops, Smartphones und Tablets an.

Während vollständig via Internet durchgeführte Interventionen in den letzten beiden Dekaden ausgiebig beforscht wurden (Hedman et al. 2012) und existierende Psychotherapiemanuale für die Anwendung im Internet übersetzt wurden, verzeichnete die Forschung um die ganzheitliche Zusammenführung von Psychotherapie und neuen Medien keine vergleichbar ausgeprägte Dynamik. Der über lange Zeit weitergeführte Einsatz von Taschencomputern (Wright et al. 2005; Przeworski und Newman 2004), die aufwendige Durchführung entsprechender Studien, die Skepsis mancher Psychotherapeuten (Becker und Jensen-Doss 2013) sowie das attraktive Einsparungspotenzial und die vielversprechende Skalierbarkeit reiner Online-Interventionen (Hedman et al. 2014) können als mögliche Gründe für diese Entwicklung vermutet werden. Obwohl die Zahl publizierter Studien bisweilen gering ist, nimmt die Beforschung gemischter Psychotherapien in den letzten Jahren zu. Dies wirft die Frage auf, ob sich die in der Online-Therapie entwickelten Programme in die Praxis klassischer Psychotherapie integrieren lassen und ob sich deren Einsatz günstig auf den Therapieprozess und die da- raus resultierenden Therapieergebnisse auswirkt.

\section{Inhalte gemischter Psycho- therapie}

\section{Beispiel 1 - E-Learning und persönliche Therapie von Depression}

Ein „Blended-treatment“-Behandlungskonzept wurde in den Niederlanden entwickelt (Kooistra et al. 2016) und basiert auf einem 16 bis 20 Einheiten umfassenden Manual kognitiver Verhaltenstherapie (Bockting und Huibers 2011). Die als intensive Kurztherapie ausgelegte Behandlung erstreckt sich über einen Zeitraum von 10 Wochen, wobei in jeder Woche eine persönliche Therapieeinheit von einer Online-Einheit gefolgt wird (•Abb. 1). Die Inhalte der Online-Einheiten werden auf einer E-Learning-Plattform bereitgestellt (Minddistrict, www.minddistrict.com) und bestehen aus videounterstützter Psychoedukation sowie Übungen, die durch Geschichten und Beispiele zweier fiktiver Patienten begleitet werden. Vom Patienten bearbeitete Übungen sind auf dem Therapeuten-Portal der E-Learning-Plattform für den Therapeuten (sowie etwaige Supervisoren) einsehbar und werden dort mit entsprechendem Feedback versehen. Stimmungs- und Aktivitätstagebücher sollen auf täglicher Basis bearbeitet und gemeinsam mit den wöchentlichen Übungen zu Beginn der jeweiligen persönlichen Einheit besprochen werden.

\section{Beispiel 2 - Smartphone- Applikation und persönliche Therapie von Depression}

Ein anderes Behandlungskonzept wurde in Schweden entwickelt (Ly et al. 2014, 2015) und basiert auf Prinzipien der Verhaltensaktivierung (Martell et al. 2001; Lejuez et al. 2001). Verhaltensaktivierung ist eine isolierte Komponente der Verhaltenstherapie, deren Fokus auf konkreten Aktivierungsstrategien liegt und die darauf abzielt, Vermeidungs- und Rückzugsverhalten zu reduzieren sowie positive Verstärkung durch wertegelei- 


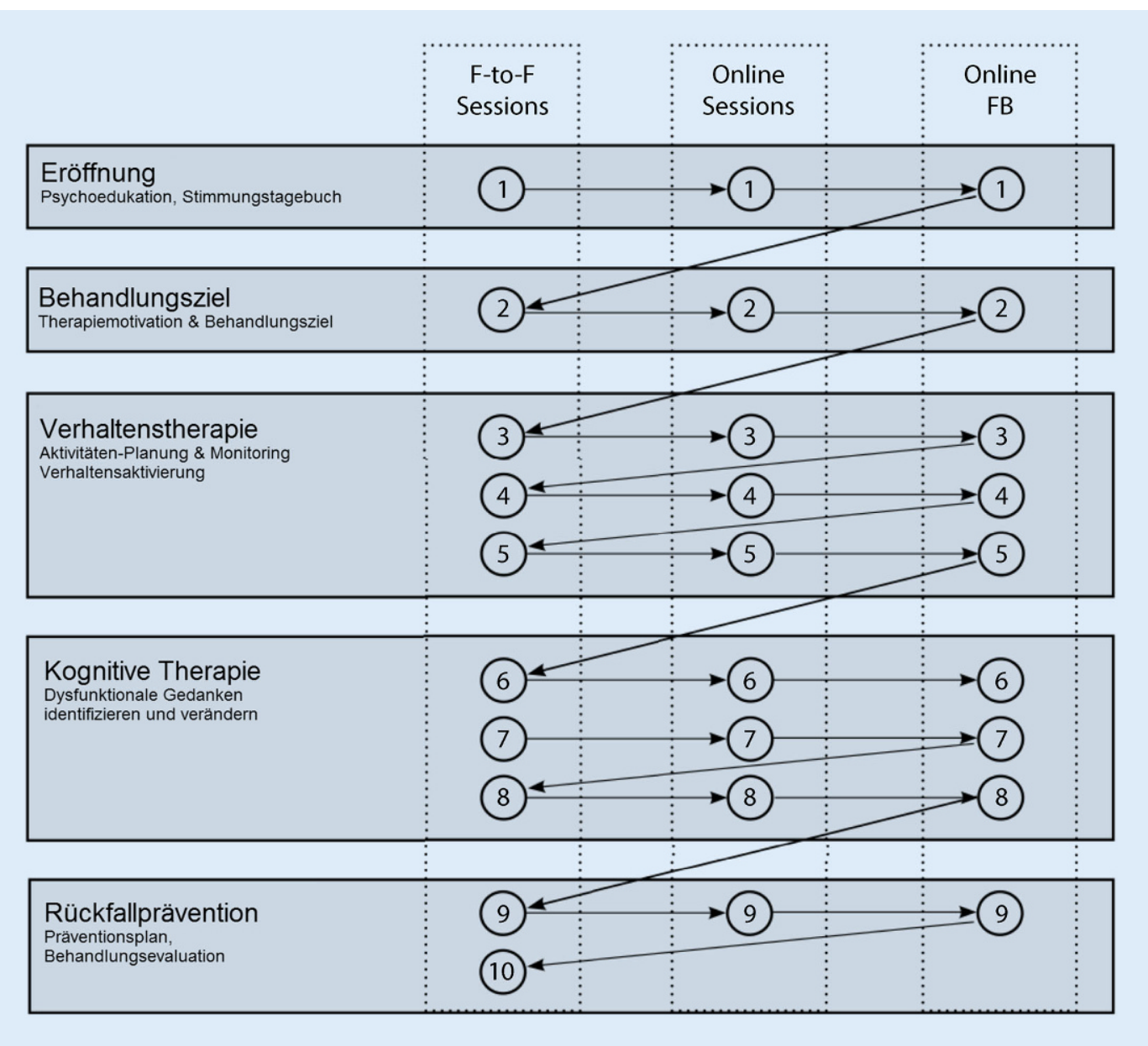

Abb. $1<$ Aufbau des "Blended-treatment" Behandlungskonzepts. F-to-F Sessions persönliche Sitzungen, Online FB Online-Feedback, 1.-10. Sitzung tetes Verhalten aufzubauen (Jacobson et al. 2001). Wegen der konkreten, am Selbstmanagement orientierten Beschaffenheit dieser Behandlungsform eignet sich dieser Ansatz besonders für eine intuitive und unkomplizierte Anwendung am Smartphone. Die Inhalte der Smartphone-App bestehen aus Psychoedukation, einer Datenbank von aktivierenden Verhaltensweisen sowie eigenen Zielen und Verhaltensweisen, die mithilfe der App definiert, verfolgt, dokumentiert, reflektiert und angepasst werden können. Therapeutische Unterstützung des Selbstmanagements erhalten Klienten in niedrigfrequenten 2-wöchentlichen persönlichen Therapiesitzungen. Die Applikation besitzt zusätzlich ein Therapeuten-Portal, über das außerhalb der Sitzungen unterstützendes Feedback oder psychoedukative Kurznachrichten an die Patienten gesendet werden.
Beispiel 3 - Computerunterstützung sowie Gruppentherapie von Angst und Depression

Weil gemischtes Lernen dem Gruppensetting entspringt, etwa als Unterstützung im universitären oder betrieblichen Kontext (Zumbach 2010), können Computer auch in der Gruppentherapie sinnvoll eingesetzt werden. Für Angststörungen wurde in einer Reihe von Untersuchungen gezeigt, dass sich Computer gut zur Unterstützung von Entspannungsübungen, kognitiver Umstrukturierung (Przeworski und Newman 2004) oder hochfrequentem Monitoring (Newman et al. 2014) eignen. Für die Behandlung von Depression liegt bisweilen nur eine Konzeptstudie vor, in der der Einsatz von E-Learning und modernen Medien (in und zwischen den Gruppensitzungen) als aktiver Wirkfaktor beschrieben wurde (Schuster et al. 2017).

\section{Empirische Befunde zu gemischter Psychotherapie}

Während die empirische Basis von Online-Interventionen als relativ fundiert bezeichnet werden kann (s. oben), befindet sich die Forschung zur gemischten Therapie erst im Aufbau. Im Sinne des Stufenmodelles der Psychotherapieforschung (Rounsaville et al. 2006) liegen Machbarkeits- und Anwendbarkeitsstudien vor, um die Akzeptanz und Durchführbarkeit gemischter Interventionen in randomisierten Studien oder unter naturalistischen Bedingungen $\mathrm{zu}$ eruieren. Gleichzeitig bestehen Bestrebungen, das klassische Therapiesetting durch Stand-alone-OnlineInterventionen flankierend $\mathrm{zu}$ unterstützen. In komparativen Studien wird gegenwärtig die Strategie verfolgt, verkürzte gemischte Therapiemanuale mit Standardmanualen der Verhaltenstherapie $\mathrm{zu}$ vergleichen, um aufzuzeigen, dass gleichwertige Therapieeffekte mit 
geringerem therapeutischen Aufwand erzielbar sind. Im Folgenden werden Beispiele für die einzelnen Forschungsstränge beschrieben.

\section{Stand-alone-Online-Intervention als Zusatz zur Psychotherapie}

Neben jenen Studien, die etwa ab Mitte der 1990er- bis etwa Mitte der 2000erJahre Taschencomputer als Adjunkt zur Unterstützung von Psychotherapie eingesetzt haben (Newman et al. 1996; Przeworski und Newman 2004), finden sich auch neuere Untersuchungen, die zeigen, dass sich der Einsatz computerisierter Verfahren positiv auf den Therapieerfolg auswirken kann. So führte etwa in einer Studie zur Behandlung von stoffgebundenen Abhängigkeiten der unterstützende Einsatz eines unabhängigen Online-Programms zu einer zusätzlichen Reduktion positiver Urinproben sowie zu längeren Abstinenzzeiten (Carroll et al. 2008). Positive Effekte konnten noch nach einem Zeitraum von 6 Monaten festgestellt werden (Carroll et al. 2009); die Ergebnisse wurden in weiteren Studien repliziert (Carroll et al. 2014). In einer groß angelegten Therapiestudie $(n=$ 800) wird aktuell die Wirksamkeit klassischer Psychotherapie mit der Wirksamkeit von Psychotherapie, die von einer unabhängigen Online-Intervention für Depression (Deprexis) begleitet wird, untersucht (Krieger et al. 2014). Stand-aloneProgramme können zusätzlich eingesetzt werden, um den Transfer und die Stabilisierung nach stationärer Psychotherapie zu fördern (Bauer et al. 2011; Ebert et al. 2013b).

\section{Machbarkeits- und Anwendbar- keitsstudien}

Therapieansätze, in denen Computermethoden tiefer in das Behandlungskonzept eingestrickt wurden, liegen v. a. im Bereich von Depression und Angststörungen vor. So untersuchten Craske et al. (2009) den Einsatz eines modularen Computerprogramms („Calm“), das von Patienten unter Beisein eines Therapeuten in gemeinsamen Sitzungen bearbeitet wurde. Die entweder an generalisierter Angststörung, sozialer
Phobie, Panikstörung oder posttraumatischer Belastungsstörung leidenden Patienten schätzten das Programm als hilfreich ein, und die guten Behandlungserfolge standen teilweise mit dem selbst eingeschätzten Verständnis der Online-Module im Zusammenhang. Das Programm wurde auch von den 13 eingebundenen Therapeuten als hilfreich bis sehr hilfreich beurteilt. Mansson et al. (2013) berichten in einer Studie zur Behandlung von Depressionen und Angst sehr hohe Behandlungseffekte und vergleichbar positive Einschätzungen der Anwendbarkeit. Zusätzlich gaben die eingebundenen Therapeuten an, dass sich der Einsatz moderner Medien positiv auf das unerwünschte Abdriften von verhaltenstherapeutischen Behandlungsmanualen auswirken könnte. Gemischte Therapieformen könnten somit dazu beitragen, empirisch fundierte Methoden und (Arbeits-)Materialien nahtloser in den Therapieprozess einfließen zu lassen.

\section{Patienten- und Therapeutensicht}

In einer mehrstufigen Delphi-Befragung unter niederländischen Patienten und Psychotherapeuten, die bereits Erfahrungen mit gemischter Psychotherapie bei der Behandlung von Depression hatten, gingen van der Vaart et al. (2014) der Frage nach dem optimalen Verschnitt computerisierter und klassischer Interventionsstrategien sowie möglicher Vor- und Nachteile gemischter Psychotherapie nach. Beide Gruppen (12 Psychotherapeuten; 8 Patienten) stimmten weitgehend darin überein, dass sich v. a. Übungen für Zuhause, Stimmungs- und Aktivitätstagebücher sowie Psychoedukation und Erinnerungsnachrichten gut über die Online-Plattform abwickeln ließen. Behandlungseröffnung und -abschluss sowie tiefergehende Gespräche über Gedanken, Gefühle und Probleme und die praktische Unterstützung bei verhaltenstherapeutischen Übungen sollten hingegen weiterhin am besten im persönlichen Setting erfolgen. Vor- und Nachteile gemischter Therapie wurden von beiden Gruppen in den Bereichen „Selbstmanagement", „therapeutische Beziehung“, „therapeutischer Prozess“ und „mögliche Risiken“ geortet. Beide Gruppen waren sich einig, dass gemischte Therapie das Selbstmanagement der Patienten fördere. Auswirkungen auf einhergehende Veränderungen einzelner Therapiesitzungen wurden insgesamt als neutral/problemlos bis positiv gewertet. Die Hälfte der Patienten äußerte jedoch Befürchtungen, dass es u. U. schwerer sein könnte, diffuse Probleme anzusprechen, und dass eine gewisse Gefahr der Schwächung der therapeutischen Beziehung bestehen könnte. Hinsichtlich möglicher Nachteile gaben Patienten an, eine gemischte Therapie sei nicht für jedermann geeignet. Therapeuten orteten die deutlichsten Nachteile in einem möglichen erhöhten Zeitaufwand bei der Durchführung der Therapie (42\%) sowie in einem gewissen Risiko für Fehlanwendungen seitens mancher Patienten (58\%). Interessant ist, dass das optimale Mischungsverhältnis von Onlineund persönlichen Komponenten für die Therapie von Patienten und Therapeuten unterschiedlich eingeschätzt wurde. So beschrieb die Mehrheit der Psychotherapeuten das optimale Mischungsverhältnis mit $50-75 \%$ persönlichem Kontaktanteil, während dieser Anteil auf Patientenseite lediglich 40-50\% betrug. Die Stärke der beschriebenen Studie liegt in der Befragung von Personen, die bereits Erfahrung im Umgang mit gemischter Therapie hatten. In der geringen Stichprobengröße der DelphiBefragung liegt hingegen die wesentlichste Einschränkung der berichteten Ergebnisse. Hinsichtlich der Indikation und der optimalen Zusammenstellung gemischter Therapie betonen Forscher derselben niederländischen Gruppe in einem Standpunktartikel die Wichtigkeit einer konsensualen Abstimmung zwischen Patienten und Therapeuten und bieten eine Checkliste zur Abschätzung der Eignung für gemischte Therapien an (Wentzel et al. 2016).

\section{Klassische und gemischte Psychotherapie im Vergleich}

Komparative Studien zwischen klassischen und gemischten Psychotherapieformen existieren bislang kaum. In einer Untersuchung von Wright et al. (2005), 
in der 8-wöchige Psychotherapie für leichte bis mittelgradige Depression mit um $50 \%$ verkürzter gemischter Therapie (25-minütige Therapiesitzungen statt $50 \mathrm{~min}$ ) verglichen wurde, ergaben sich vergleichbare Prä-post-Effekte $(d=1,04$ für das klassische Format und $d=1,14$ für das verkürzte Format). Die Abbrecherquote lag mit $13 \%$ im normalen Bereich, und die Ergebnisse einer Vorgängerstudie ergaben hohe Zufriedenheit mit dem computerunterstützten Therapieformat (Wright et al. 2002). Ähnliche Ergebnisse erzielten Ly et al. (2015) mit dem von ihnen entwickelten gemischten Konzept zur Behandlung von Depressionen (Verhaltensaktivierung).

Gegenwärtig werden gleich mehrere Vergleichsstudien für Depression und Angststörungen mit ähnlichem Versuchsaufbau durchgeführt. Dabei werden typischerweise 15- bis 20-stündige Verhaltenstherapien (oder „Treatmentas-usual“"TAU]-Bedingungen) mit gemischten Therapien verglichen, in denen die Hälfte der Sitzungen alternierend als selbstständig zu bearbeitende Onlinesitzungen zu bewältigen sind (Romijn et al. 2015; Kemmeren et al. 2016). Manche dieser Studien (Kemmeren et al. 2016) bilden einen Teil des Projektes „European Comparative Effectiveness research on blended Depression treatment versus treatment-as-usual“ (E-COMPARED), einer 2014 ins Leben gerufenen europäischen Forschungsinitiative aus 8 Ländern, die gemischte Psychotherapie in der Regelversorgung untersuchen soll (Kleiboer et al. 2016). Alle Studien verfolgen das Ziel, gleiche Behandlungsergebnisse trotz umfangreicher Reduktion des direkten therapeutischen Face-to-faceAnteils zu erzielen. Obwohl die Intensivierung psychotherapeutischer Behandlungen als möglicher Vorteil gemischter Therapie genannt wird (Kooistra et al. 2016), liegt bisweilen keine kontrollierte randomisierte Studie vor, die das Ziel verfolgt, bessere Behandlungsergebnisse unter Gleichhaltung des therapeutischen Arbeitsaufwandes zu erzielen. Die weiter oben bereits angesprochene groß angelegte Studie von Krieger et al. (2014), in der eine Stand-alone-Online-Intervention als Zusatz zur Psychotherapie untersucht werden soll, kommt diesem Ansinnen am nächsten.

\section{Erprobung gemischter Therapie in der Regelversorgung}

Der Einsatz gemischter Therapie in der Regelversorgung wurde in den Jahren 2009 bis 2012 in einer naturalistischen Kosteneffektivitätsstudie in den Niederlanden untersucht (Kenter et al. 2015). Von den über 4000 in diesem Zeitraum behandelten Patienten wurden 168 an einer Depression oder Angststörung leidende Patienten für die Untersuchung ausgewählt und mit einer parallelisierten Vergleichsgruppe gematcht. Die OnlineKomponenten der Behandlungen bestanden aus bedarfsgerecht applizierten störungsspezifischen Modulen, wie etwa „Achtsamkeit“, „Gedanken und Gefühle“, „Gefühle und Verhalten“, „Verhaltensübungen“, „Exposition“, „Panikmanagement" oder „Rückfallprophylaxe“. Entgegen den Erwartungen führte die gemischte Psychotherapie weder zu einer Kostenreduktion noch zu besseren Therapieerfolgen. Vielmehr erhöhten sich die Kosten pro behandeltem Patienten im Schnitt um 45-50\%, wobei sich der Zuwachs überwiegend aus einer Erhöhung der Gesamttherapiedauer ergab. So belief sich die durchschnittliche Anzahl an persönlichen Therapiesitzungen bei Angstpatienten in beiden Gruppen gleichermaßen auf 12 Einheiten, wohingegen in der gemischten Therapiegruppe im Schnitt zusätzlich noch 11 Online-Einheiten hinzukamen. Die Online-Einheiten flossen zum normalen Stundensatz für Psychotherapie in die Kostenrechnung ein. Zur Erfassung des Therapieerfolgs wurde die Globale Erfassung des Funktionsniveaus (GAFIndex) nach DSM-IV herangezogen. Obwohl der GAF-Index gelegentlich als klinisches Fremdbeurteilungsverfahren zur Abbildung von Therapieerfolgen herangezogen wird, sind sein Einsatz und der Verzicht auf störungsspezifische Symptomskalen sowie das Fehlen der exakten Kosten der Online-Behandlung als wichtige Schwächen der Studie zu bezeichnen. Die Autoren betonen die Notwendigkeit einer umfassenden Mitarbeiterschulung, um die zweckmäßige
Anwendung der Online-Module zu gewährleisten, und argumentieren, dass ein etwaiger therapeutischer Mehraufwand v. a. die Phase der Umstellung von klassischer zu gemischter Psychotherapie betreffe.

\section{Gemischte Therapie in der Praxis}

Obwohl Psychotherapeuten E-Mail-Korrespondenz und Mobiltelefonie für organisatorische Zwecke nutzen (Eichenberg und Kienzle 2011), ist der Einsatz gemischter Psychotherapie in der Regelversorgung im deutschen Sprachraum unüblich. In diesem Bereich nehmen die Niederlande wiederum eine europäische Vorreiterrolle ein. Nach Angaben des führenden Software-Anbieters (minddistrict) beläuft sich die Anzahl therapeutischen Fachpersonals, das die SoftwareDienste des Anbieters zurzeit regelmäßig nutzt, auf 17.090 Professionisten, und die Zahl aktiv mit der Plattform arbeitender Patienten betrug zum Stichtag (29. September 2016) 126.277 Patienten.

\section{Zusammenschau und Ausblick}

Gemeinsam mit der Entwicklung, die moderne Medien und Kommunikationsformen gegenwärtig in der Gesellschaft durchlaufen, ist auch im psychosozialen Sektor sowie im Bereich der Onlineund gemischten Psychotherapien in den letzten Jahren vieles in Bewegung gekommen. Angesichts der weiterwachsenden Leistungsfähigkeit von Computern und Mobiltelefonen sowie dem fortschreitenden Anwendungswissen ihrer User scheint dieser Trend den gegenwärtigen Zeitgeist widerzuspiegeln. Die empirische Basis zu Nutzbarkeit und Wirksamkeit von Online-Interventionen wächst und wird zunehmend verlässlicher. In den letzten Jahren entstanden entsprechend erste Online-Kliniken in Europa und Australien. Die dabei gewonnenen Daten besitzen bereits hohe Aussagekraft über die zu erwartende Wirkung von Online-Interventionen in der Regelversorgung (Hedman et al. 2014). Entsprechend werden auf Ebene der Europäischen Union (EU) gegenwärtig mehrere groß angelegte Anwendungsund Kosteneffektivitätsstudien geför- 
dert (etwa iCARE, www.icare-online. eu; MasterMind, www.mastermindproject.eu oder E-COMPARED, www. e-compared.eu).

In der Analyse und Zusammenschau der Situation dürfen allerdings kritische Stimmen nicht ungehört bleiben. So bezeichnen solche die Erwartung, OnlineProgramme könnten dabei helfen, bestehende Versorgungslücken zu schließen, als überzogen und warnen vor einer fortschreitenden Ökonomisierung der Psychotherapie und einer zunehmenden Fragmentierung der Therapeut-PatientBeziehung (Apolinário-Hagen und Tasseit 2015). Mit einem nüchternen Blick auf die gegenwärtige Forschungssituation ist festzuhalten, dass die meisten Studien ein sehr eingeschränktes Repertoire an Störungen (primär depressive, Angst- und Belastungsstörungen) untersucht haben. Einschränkungen in der Anwendung von Online-Interventionen bestehen dementsprechend sowohl auf Ebene verschiedener differenzieller Aspekte und spezifischer Problembereiche (z. B. Suizidalität, Intelligenzminderung, psychotische Störungen) als auch hinsichtlich demografischer Merkmale (etwa Alter, Bildungsstand, Internetkompetenz etc.) sowie hinsichtlich persönlicher Präferenzen (Tarrier et al. 2006; Andersson und Titov 2014) und gesetzlicher Rahmenbedingungen (Bauer und Kordy 2008). Umfragestudien zeigen, dass Patienten, die sich auf einer Warteliste für Psychotherapie befinden, ein geringes Interesse an einer Versorgung durch Online-Interventionen besitzen (Gieselmann et al. 2015). Dieser Befund besitzt v. a. für den „Stepped-care“-Ansatz (Nordgreen et al. 2015), also jenem Versorgungsmodell, in dem Online-Programme gestuft als erster Interventionsschritt eingesetzt werden sollen, besondere Relevanz. Zusätzlich scheint der Faktor Mensch in Online-Interventionen als wesentliche Erfolgskomponente unersetzbar zu bleiben. So wie ein Selbsthilfebuch jederzeit zur Seite gelegt werden kann, dürfte es auch bei Online-Interventionen wichtig sein, ein Gegenüber zu haben, dem man sich öffnen kann und das durch die Herstellung einer tragfähigen therapeutischen Beziehung unterstützend auf die erfolgreiche Durchführung des OnlineProgramms hinwirkt.

Die von der Forschung entwickelten Online-Programme lassen sich jedoch auch in die persönliche Psychotherapie von Angesicht zu Angesicht integrieren. Hierbei sind viele Kombinationen beider Behandlungsstrategien möglich. Das deklarierte Ziel gemischter Psychotherapie ist es, das Beste aus beiden Welten vereinen zu wollen (Ruwaard und Kok 2015). So bleiben der persönliche Kontakt und die therapeutische Beziehung in ihrer bewährten Form erhalten, während OnlineKomponenten (basierend aufE-Learning und Anwendungen für Mobiltelefone) flankierend zur Verbesserung des Therapieprozesses beitragen können. Erste Befunde weisen auf eine gute Akzeptanz gemischter Therapie hin, wobei Therapeuten und Patienten gleichermaßen Vorteile hinsichtlich eines verbesserten Selbstmanagements sehen (Van der Vaart et al. 2014). Auch Akteure des Gesundheitswesens und politische Entscheidungsträger scheinen gemischten Psychotherapieansätzen gegenüber positiver eingestellt zu sein (Topooco et al. 2017) und diesem Ansatz besondere Attraktivität zuzuschreiben (Ruwaard und Kok 2015). Der Grund hierfür dürfte in der guten Eingliederbarkeit in bestehende Versorgungsstrukturen liegen.

Gleichzeitig gibt es aber auch hierzu einige kritische Punkte einzubringen: Erste naturalistische Studien zeigen einige Schwachstellen gemischter Interventionen: So könnten Patienten OnlineKomponenten etwa falsch oder exzessiv anwenden, und eine zu starke Straffung des Therapieprozesses könnte negative Effekte auf tiefer liegende oder weniger greifbare Therapieanliegen nach sich ziehen (Van der Vaart et al. 2014). Obwohl gemischte Therapie durch eine Intensivierung der Behandlung das Potenzial besitzt, bestehende Psychotherapie wirkungsvoll zu ergänzen, zielen die meisten gegenwärtigen Studien auf eine Verringerung der Therapiedauer ab. Somit scheinen besonders in diesem Bereich ökonomische Überlegungen Priorität zu besitzen. Die Sinnhaftigkeit dieser Strategie muss noch weiter beforscht werden, denn erste Studien zeigen, dass die Hinzufügung von Onlinekomponenten die
Therapie nicht notwendigerweise kürzer und effektiver macht (Kenter et al. 2015). Zudem warnen niederländische Forscher unter dem Motto „Wild West eHealth“ vor einer allzu raschen und vordergründig wirtschaftlich motivierten Anwendung gemischter Therapien in der Regelversorgung, die eine indirekte Ausdünnung gängiger Therapiestandards zur Folge haben könnte (Ruwaard und Kok 2015). Die eingeforderte Validierung gemischter Psychotherapie in der Regelversorgung bedarf nicht nur in den Niederlanden ambitionierter Anstrengungen, um der Anwendung ungeprüfter SoftwareAngebote privater Kliniken und Versicherer nicht nachzustehen.

Zusammengefasst scheinen sowohl Online-Interventionen als auch gemischte Psychotherapie vielseitig und sinnvoll in der psychosozialen Versorgung einsetzbar zu sein. Die Integration moderner Medien in bestehende psychotherapeutische Behandlungsformen („blended therapy“) könnte einen Trend widerspiegeln, der in anderen Gesellschaftsbereichen bereits nachhaltig Einzug gehalten hat (etwa: „blended learning“ in der betrieblichen Weiterbildung oder E-Learning in der universitären Ausbildung). Der Einsatz reiner internetbasierter Interventionen zur Behandlung häufiger psychischer Beeinträchtigungen in der Regelversorgung wird gegenwärtig international erfolgreich erprobt und dürfte über kurz oder lang Vorbildwirkung für Gesundheitssysteme im deutschen Sprachraum haben. Einer klaren begrifflichen und gesetzlichen Trennung zwischen klassischer Psychotherapie und Online-Interventionen sowie einer umfassenden Evaluierung eingesetzter Programme sollte daher besonderer Stellenwert beigemessen werden.

\section{Fazit für die Praxis}

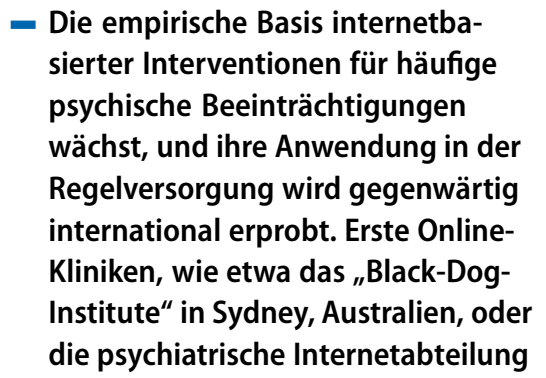


am Karolinska-Universitätskrankenhaus in Stockholm wurden bereits ins Leben gerufen. Diese Entwicklung dürfte mittel- bis langfristig Vorbildwirkung für die Gesundheitssysteme im deutschen Sprachraum besitzen.

- In vielen Studien steht therapeutische Begleitung mit besseren Behandlungserfolgen und niedrigeren Abbruchquoten in Verbindung. Eine zufriedenstellende therapeutische Allianz scheint bei internetbasierten Interventionen realisierbar zu sein. Hinsichtlich der Anwendung internetbasierter Interventionen bestehen jedoch Einschränkungen.

- In der gemischten Therapie (,blended therapy") wird klassische Psychotherapie von modernen Medien unterstützt. Dabei lautet das deklarierte Ziel, Vorteile beider Behandlungsformen vereinen zu wollen.

- Erste empirische Befunde sprechen für eine gute Anwendbarkeit gemischter Therapie im Einzel- und im Gruppensetting. Die Niederlande nehmen eine Vorreiterrolle in der Dissemination gemischter Therapie ein.

- Ob diese Behandlungsform auch wirtschaftliches Einsparungspotenzial besitzt, wird gegenwärtig in dem multilateralen Forschungsprojekt E-COMPARED untersucht.

\section{Korrespondenzadresse}

\section{R. Schuster, MSc.}

Fachbereich Psychologie, Universität Salzburg Hellbrunnerstr. 34, 5020 Salzburg, Österreich raphael.schuster@sbg.ac.at

Acknowledgements. Open access funding provided by Paris Lodron University of Salzburg.

\section{Einhaltung ethischer Richtlinien}

Interessenkonflikt. R. Schuster, T. Berger und A.-R. Laireiter geben an, dass kein Interessenkonflikt besteht.

Dieser Beitrag beinhaltet keine von den Autoren durchgeführten Studien an Menschen oder Tieren.

Open Access. Dieser Artikel wird unter der Creative Commons Namensnennung 4.0 International Lizenz (http://creativecommons.org/licenses/by/4.0/deed. de) veröffentlicht, welche die Nutzung, Vervielfäl- tigung, Bearbeitung, Verbreitung und Wiedergabe in jeglichem Medium und Format erlaubt, sofern Sie den/die ursprünglichen Autor(en) und die Quelle ordnungsgemäßnennen, einen Linkzur Creative Commons Lizenz beifügen und angeben, ob Änderungen vorgenommen wurden.

\section{Literatur}

Adelman CB, Panza KE, Bartley CA et al (2014) A metaanalysis of computerized cognitive-behavioral therapy for the treatment of DSM-5 anxiety disorders. JClin Psychiatry 75:e695-e704

Andersson G (2014) The internet and CBT: a clinical guide. CRCPress, Boca Raton, $\mathrm{S} 2$

Andersson G, Titov N (2014) Advantages and limitations of Internet-based interventions for common mental disorders. World Psychiatry 13:4-11

Andersson G, Paxling B, Wiwe M et al (2012) Therapeutic alliance in guided internet-delivered cognitive behavioural treatment of depression generalized anxiety disorder and social anxiety disorder. Behav Res Ther 50:544-550

Andersson G, Cuijpers P, Carlbring P et al (2014) Guided internet-based vs. face-to-face cognitive behavior therapy for psychiatric and somatic disorders: a systematic review and metaanalysis. World Psychiatry 13:288-295

Andrews G, Williams AD (2014) Up-scaling clinician assisted internet cognitive behavioural therapy (iCBT) for depression: a model for dissemination into primary care. Clin Psychol Rev 41:40-48

Apolinário-Hagen J, Tasseit S (2015) Chancen und Risiken der Internettherapie für die Regelversorgung in Deutschland. Ein Beitrag zur Psychotherapie im Zeitalter von Web 2.0. E-Beratungsjournal 11:69-86

Baer L, Surman OS (1985) Microcomputer-assisted relaxation. Percept Mot Skills 61:499-502

Barak A, Klein B, Proudfoot JG (2009) Defining Internetsupported therapeutic interventions. Ann Behav Med 38:4-17

Bauer S, Kordy H (2008) E-Mental-Health - Neue Medien in der psychosozialen Versorgung. Springer, Heidelberg, S13-17

Bauer S, Wolf M, Haug S, Kordy H (2011) The effectiveness of internet chat groups in relapse prevention after inpatient psychotherapy. Psychother Res 21:219-226

Becker EM, Jensen-Doss A (2013) Computer-assisted therapies: examination of therapist-level barriers to their use. Behav Ther 44:614-624

Bendelin N, Hesser H, Dahl J et al (2011) Experiences of guided Internet-based cognitive-behavioural treatment for depression: a qualitative study. BMCPsychiatry 11:107

Berger T (2015) Internetbasierte Interventionen bei psychischen Störungen. Fortschritte der Psychotherapie, Bd. 57. Hogrefe, Göttingen, S36-38

Berger T (2016) The therapeutic alliance in internet interventions: a narrative review and suggestions for future research. Psychother Res 6:1-14

Berger T, Caspar F (2011) Internetbasierte Psychotherapien. Psych Up2date 5:29-43

Berger T, Caspar F, Richardson R et al (2011) Internetbased treatment of social phobia: a randomized controlled trial comparing unguided with two types of guided self-help. Behav Res Ther 48:158-169

Bergman NL, Nordgren L, Carlbring P et al (2013) Role of the working alliance on treatment outcome in tailored internet-based cognitive behavioural therapy for anxiety disorders: randomized controlled pilot trial. JMIR Res Protoc 2:e4

Bockting CLH, Huibers MJH (2011) Treatment protocol for patients with a depressive disorder Protocollaire behandeling van patienten met een depressieve stoornis. In: Keijsers GPJ, Van Minnen A, Hoogduin CAL (Hrsg) Protocollaire behandelingen voor volwassenen met psychische klachten. Uitgeverij Boom, Amsterdam, S251-288

Carlbring P, Smit F (2008) Randomized trial of internetdelivered self-help with telephone support for pathological gamblers. J Consult Clin Psychol 76:1090-1094

Carrard I, Crépin C, Rouget P et al (2011) Randomised controlled trial of a guided self-help treatment on the internet for binge eating disorder. Behav Res Ther 49:482-491

Carroll K, Ball S, Martino S et al (2008) Computerassisted delivery of cognitive-behavioral therapy for addiction: a randomized trial of CBT4CBT. Am JPsychiatry 165:881-888

Carroll KM, Ball SA, Martino $S$ et al (2009) Enduring effects of a computer-assisted training program for cognitive behavioral therapy: a 6-month follow-up of CBT4CBT. Drug Alcohol Depend 100:178-181

Carroll KM, Kiluk BD, Nich C et al (2014) Computerassisted delivery of cognitive-behavioral therapy: efficacy and durability of CBT4CBT among cocaine-dependent individuals maintained on methadone. Am J Psychiatry 171:436-444

Craske MG, Rose RD, Lang A et al (2009) Computer assisted delivery of cognitive behavioral therapy for anxiety disorders in primary care settings. Depress Anxiety 26:235-242

Donkin L, Glozier N (2012) Motivators and motivations to persist with online psychological interventions: a qualitative study of treatment completers. J Med Internet Res 14:e91

Ebert DD, Gollwitzer M, Riper H et al (2013a) For whom does it work? Moderators of outcome on the effect of a transdiagnostic internetbased maintenance treatment after inpatient psychotherapy: randomized controlled trial. JMed Internet Res 15:e191

Ebert DD, Tarnowski T, Gollwitzer M et al (2013b) A transdiagnostic internet-based maintenance treatment enhances the stability of outcome after inpatient cognitive behavioral therapy: a randomized controlled trial. Psychother Psychosom 82:246-256

Ebert DD, Lehr D, Baumeister H et al (2014) GET.ON Mood Enhancer: efficacy of Internet-based guided self-help compared to psychoeducation for depression: an investigator-blinded randomised controlled trial. Trials 15:39

Eells TD, Wright JH, Barrett MS et al (2014) Computerassisted cognitive - behavior therapy for depression. Psychotherapy (Chic) 51:191-197

Eichenberg C, Kienzle K(2011) Psychotherapeuten und Internet. Psychotherapeut 11:485-493

Eichenberg C, Wolters C, Brähler E (2013) The Internet as a mental health advisor in Germany - results of a national survey. PLOSONE 8:e79206

Farrer LM, Griffiths KM, Christensen H et al (2013) Predictors of adherence and outcome in Internet-based cognitive behavior therapy delivered in a telephone counseling setting. Cognit Ther Res 27:358-367

Gerhards SAH, Abma TA, Arntz A et al (2011) Improving adherence and effectiveness of computerised cognitive behavioural therapy without support 
for depression: a qualitative study on patient experiences. J Affect Disord 129:117-125

Gieselmann A, Böckermann M, Pietrowsky R (2015) Internetbasierte Gesundheitsinterventionen. Evaluation aus der Perspektive von Patienten vor und während ambulanter Psychotherapie. Psychotherapeut 60:433-440

Hedman E, Ljotsson B, Lindefors N (2012) Cognitive behavior therapy via the Internet: a systematic review of applications, clinical efficacy and cost-effectiveness. Expert Rev Pharmacoecon Outcomes Res 12:745-764

Hedman E, Ljotsson B, Kaldo V et al (2014) Effectiveness of Internet-based cognitive behaviour therapy for depression in routine psychiatric care. J Affect Disord 55:49-58

Horvath AO, Greenberg LS (1989) Development and validation of the Working Alliance Inventory. JCouns Psychol 36:223

Jacobson NS, Martell CR, Dimidjian S (2001) Behavioral activation treatment for depression: returning to contextual roots. Clin Psychol Sci Pract 8:255-270

Johansson R, Andersson G (2012) Internet-based psychological treatments for depression. Expert Rev Neurother 12:861-870

Johansson R, Ekbladh S, Hebert A et al (2012) Psychodynamic guided self-help for adult depression through the Internet: a randomised controlled trial.PLOSONE 7:e38021

Kemmeren LL, van Schaik DJ, Riper $\mathrm{H}$ et al (2016) Effectiveness of blended depression treatment for adults in specialised mental healthcare: study protocol for a randomised controlled trial. BMC Psychiatry 16:113

Kenter RMF, van de Ven PM, Cuijpers P et al (2015) Costs and effects of Internet cognitive behavioral treatment blended with face-to-face treatment: results from a naturalistic study. Invent 2:77-83

Kleiboer A, Smit J, Bosmans J et al (2016) European COMPARative Effectiveness research on blended Depression treatment versus treatment-as-usual (E-COMPARED): study protocol for a randomized controlled, non-inferiority trial in eight European countries. Trials 17:387

Klein JP, Berger T, Schröder J et al (2016) Effects of a psychological Internet intervention in the treatment of mild to moderate depressive symptoms: results of the EVIDENT study, a randomized controlled trial. Psychother Psychosom 85:218-228

Knaevelsrud C, Maercker A (2006) Does the quality of the working alliance predict treatment outcome in online psychotherapy for traumatized patients? J Med Internet Res 8:e31

Kooistra LC, Ruwaard J, Wiersma JE et al (2016) Development and initial evaluation of blended cognitive behavioural treatment for major depression in routine specialized mental health care. Invent 4:61-71

Krieger T, Meyer B, Sude K et al (2014) Evaluating an e-mental health program ("deprexis") as adjunctive treatment tool in psychotherapy for depression: design of a pragmatic randomized controlled trial. BMC Psychiatry 14:285

Kuester A, Niemeyer H, Knaevelsrud C (2016) Internetbased interventions for posttraumatic stress: a meta-analysis of randomized controlled trials. Clin Psychol Rev 43:1-16

Lambert MJ, Barley DE (2002) Research summary on the therapeutic relationship and psychotherapy outcome. In: Norcross JC (Hrsg) Psychotherapy relationships that work. Oxford University Press, Oxford, S17-32
Lejuez CW, Hopko DR, Hopko SD (2001) A brief behavioral activation treatment for depression. Treatment manual. Behav Modif 25:255-286

Lin J, Lüking M, Ebert DD et al (2014) Effectiveness and cost-effectiveness of a guided and unguided internet-based acceptance and commitment therapy for chronic pain: study protocol for a three-armed randomised controlled trial. Invent 2:7-16

Ly KH, Trüschel A, Jarl L et al (2014) Behavioura activation versus mindfulness-based guided self-help treatment administered through a smartphone application: a randomised controlled trial. BMJ Open 4:e003440

Ly KH, Topooco N, Cederlund H et al (2015) Smartphone-supported versus full behavioural activation for depression: a randomised controlled trial. PLOSONE 10:e0126559

MacLeod M, Martinez R, Williams C (2009) Cognitive behaviour therapy self-help: who does it help and what are its drawbacks? Behav Cogn Psychother 37:61-72

Martell CR, Addis ME, Jacobson NS (2001) Depression in context: strategies for guided action. Norton New York

Meyer B, Berger T, Caspar F et al (2009) Effectiveness of a novel integrative online treatment for depression (Deprexis): randomized controlled trial. J Med Internet Res 11:e15

Meyer B, Bierbrodt J, Schröder J et al (2015) Effects of an Internet intervention (Deprexis) on severe depression symptoms: randomized controlled trial. Invent 2:48-59

Månsson KN, Skagius Ruiz E, Gervind E et al (2013) Development and initial evaluation of an Internet-based support system for face-to-face cognitive behavior therapy: a proof of concept study. J Med Internet Res 15:e280

Månsson KNT, Salami A, Frick A et al (2016) Neuroplasticity in response to cognitive behavior therapy for social anxiety disorder. TransI Psychiatry 6:e727

Newman MG, Kenardy J, Herman S, Taylor CB (1996) The use of hand-held computers as an adjunct to cognitive-behavior therapy. Comput Human Behav 12:135-143

Newman MG, Przeworski A, Consoli A et al (2014) $A$ randomized controlled trial of ecological momentary intervention plus brief group therapy for generalized anxiety disorder. Psychotherapy (Chic) 51:198

Nordgreen T, Haug T, Öst LG et al (2015) Stepped care versus direct face-to-face cognitive behavior therapy for social anxiety disorder and panic disorder: a randomized effectiveness trial. Behav Ther 47:166-183

Orlinsky DE, Ronnestad MH, Willutzki U (2004) Fifty years of psychotherapy process-outcome research: Continuity and change. In: Lambert MJ (Ed) Bergin and Garfield's handbook of psychotherapy and behavior change. Wiley, New York, 307-389

Paxling B, Lundgren S, Norman A et al (2013) Therapist behaviours in Internet-delivered cognitive behaviour therapy: analyses of e-mail correspondence in the treatment of generalized anxiety disorder. Behav Cogn Psychother 41:280-289

Preschl B, Maercker A, Wagner B (2011) The working alliance in a randomized controlled trial comparing online with face-to-face cognitive behavioral therapy for depression. BMC Psychiatry 11:189

Przeworski A, Newman MG (2004) Palmtop computerassisted group therapy for social phobia. J Clin Psychol 60:179-188
Richards D, Richardson T (2012) Computer-based psychological treatments for depression: a systematic review and meta-analysis. Clin Psychol Rev 32:329-342

Riper H, van Ballegooijen W, Kooistra LC et al (2013) Prevention \& eMental-health - preventie \& eMental-health. Vrije Universiteit, Amsterdam

Robinson A (2015) Meet Ellie, the machine that can detect depression. The Guardian. https:// www.theguardian.com/sustainable-business/ 2015/sep/17/ellie-machine-that-can-detectdepression.Zugegriffen: 17.Nov. 2015

Romijn G, Riper H, Kok Ret al (2015) Cost-effectiveness of blended vs. face-to-face cognitive behavioural therapy for severe anxiety disorders: study protocol of a randomized controlled trial. BMC Psychiatry 12:311

Rounsaville BJ, Carrol KM, Onken LS (2006) A stage model of behavioral therapies research: getting started and moving on from stage I. Clin Psychol Sci Pract 8:133-142

Ruwaard J, KokR (2015) Wild west eHealth: time to hold our horses? Eur Health Psychol 17:45-49

Schuster R, Leitner I, Carlbring P, Laireiter AR (2017) Exploring blended group interventions for depression: Randomised controlled feasibility study of a blended computer- and multimediasupported psychoeducational group intervention for adults with depressive symptoms. Invent 8:63-71

Suler J (2004) The online disinhibition effect. Cyberpsychol Behav 7:321-326

Tarrier N, Liversidge T, Gregg L (2006) The acceptability and preference for the psychological treatment of PTSD. Behav Res Ther 44:1643-1656

Titov N, Dear BF, Staples LG et al (2015) Disorderspecific versus transdiagnostic and clinicianguided versus self-guided treatment for major depressive disorder and comorbid anxiety disorders: arandomized controlled trial.J Anxiety Disord 35:88-102

Topooco N, Riper H, Araya R et al (2017) Attitudes towards digital treatment for depression: a European stakeholder survey. Invent 8:1-9

Tossmann HP, Jonas B, Tensil MD et al (2011) A controlled trial of an internet-based intervention program for cannabis users. Cyberpsychol Behav Soc Netw 14:673-679

Van der Vaart R, Witting M, Riper Het al (2014) Blending online therapy into regular face-to-face therapy for depression: content, ratio and preconditions according to patients and therapists using a Delphi study. BMCPsychiatry 14:355

Wagner B, Knaevelsrud C, Maercker A (2006) Internetbased cognitive-behavioral therapy for complicated grief: a randomized controlled trial. Death Stud 30:429-453

Waller R, Gilbody S (2009) Barriers to the uptake of computerized cognitive behavioural therapy: a systematic review of the quantitative and qualitative evidence. Psychol Med 39:705-712

Watkins PL (2008) Self-help therapies: pastand present. In: Watkins PL, Clum GA (Hrsg) Handbook of selfhelp therapies. Routledge, New York, S1-24

Weizenbaum J (1966) Eliza - a computer program for study of natural language communication between man and machine. Commun ACM 9:36-45

Wentzel J, van der Vaart R, Bohlmeijer ET et al (2016) Mixing online and face-to-face therapy: how to benefit from blended care in mental health care. JMIR Ment Health 3:e9

Wright JH, Wright AS, Salmon P et al (2002) Development and initial testing of a multimedia program 


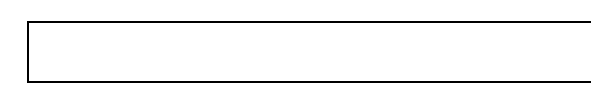

for computer-assisted cognitive therapy. Am J Psychother 56:76-86

Wright JH, WrightAS, Albano AMetal (2005)Computerassisted cognitive therapy for depression: maintaining efficacy while reducing therapist time. Am J Psychiatry 162:1158-1164

Zumbach J (2010) Lernen mit neuen Medien. Instruktionspsychologische Grundlagen. Kohlhammer, Stuttgart

\section{Fachnachrichten}

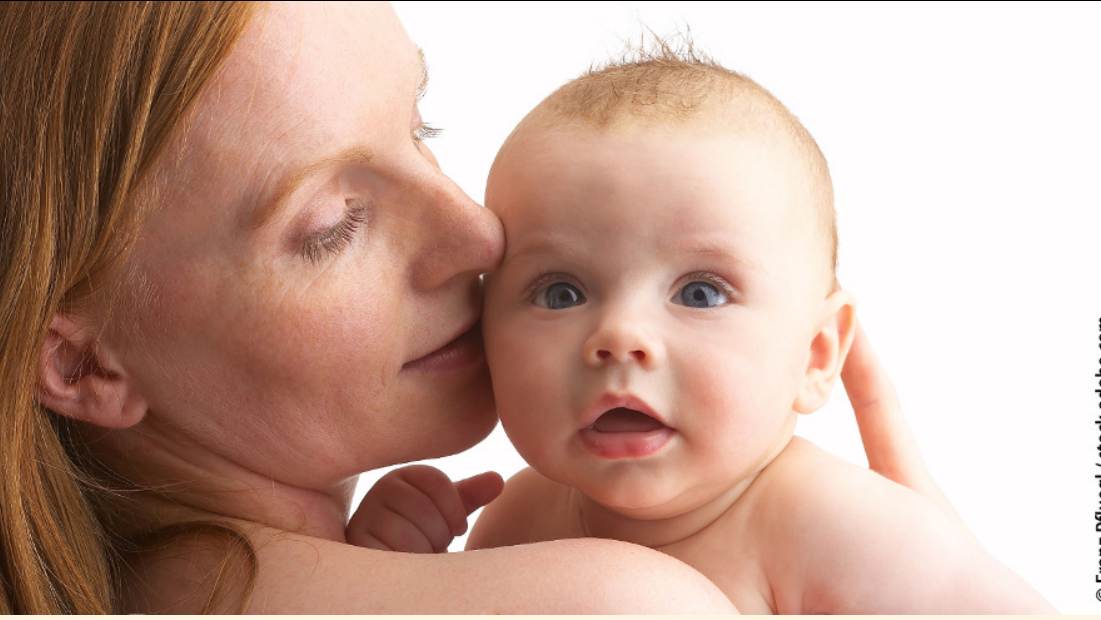

\section{Mutter-Kind-Bindung - Oxytocin schlägt Testosteron}

Hormone beeinflussen die mütterlichen Gefühle. Während Oxytocin die Bindung zum Kind stärkt, scheint Testosteron mütterliches Verhalten zu unterdrücken. Eine aktuelle Studie zeigt nun, dass Oxytocin die negative Wirkung von Testosteron ausgleichen kann.

Große Augen, Stupsnase und Pausbacken sind typische Merkmale von Kindergesichtern. Sie wirken als Schlüsselreiz im Belohnungssystem des Gehirns und bringen die Eltern dazu, sich um den Nachwuchs zu kümmern. Auch die Sexualhormone Oxytocin und Testosteron beeinflussen dieses Belohnungssystem im Hirn. Oxytocin stärkt die soziale Bindung der Mutter zum Kind; Testosteron dagegen wirkt vermutlich negativ auf mütterliche Verhaltensweisen.

In einer Studie mit 57 Studentinnen haben Wissenschaftler nun untersucht, wie die beiden Hormone interagieren und ob sie Reaktionen auf das Kindchenschema beeinflussen. Dafür teilte sie die Probandinnen in zwei Gruppen ein: Der ersten Gruppe wurde vor dem Test Oxytocin verabreicht, die zweite Gruppe bekam ein Placebo. Bei allen Versuchsteilnehmerinnen wurde zudem der Testosteronspiegel gemessen. Anschließend analysierten die Forscher mithilfe der funktionellen Magnetresonanztomographie (fMRT) die Hirnaktivität der Studienteilnehmerinnen, während diese sich Bilder von Erwachsenen und Kindern mit unterschiedlich ausgeprägtem Kindchenschema ansahen. Das Ergebnis: Erhielten Frauen mit hohen Testosteronspiegeln Oxytocin, erhöhte sich die Aktivität im Belohnungssystem des Gehirns, sobald sie Babygesichter sahen. Außerdem reagierte die Frauengruppe nach der Gabe von Oxytocin deutlicher sensitiver auf das Kindchenschema. Bei Teilnehmerinnen, die ein Placebo bekommen hatten, zeigte sich keine erhöhte Präferenz.

Davon ausgehend vermuten die Forscher, dass Oxytocin die negative Wirkung von Testosteron ausgleichen und das Fürsorgeverhalten der Frau motivieren kann. Bei Frauen mit niedrigen Testosteronwerten hatte das verabreichte Oxytocin übrigens keine Wirkung. Niedrige Werte des männlichen Sexualhormons führen also evtl. dazu, dass Frauen ein mütterliches Verhalten entwickeln und ein zusätzlicher Einfluss von Oxytocin nicht notwendig ist.

\section{Originalpublikation:}

Holtfrerich SKC, Pfister R, El Gammal AT et al. (2018): Endogenous testosterone and exogenous oxytocin influence the response to baby schema in the female brain; Scientific Reports, DOI: 10.1038/s41598-018-26020-4

Quelle: Universität Hamburg (www.unihamburg.de) 